\title{
Differential sensitivity of radionuclide ventriculography for the detection of anterior and inferior infarction
}

\author{
S R UNDERWOOD, S WALTON, P J LAMING, P J ELL, R W EMANUEL, \\ R H SWANTON \\ From the Middlesex Hospital and Medical School, London
}

SUMMARY Attenuation of counts from the more distant inferior portion of the left ventricular blood pool in equilibrium radionuclide ventriculography may mean that inferior infarction is less likely to be detected than anterior infarction. Fourier amplitude and phase images can be used to map the extent and timing of regional ventricular wall motion and this study assesses their use for the detection of anterior and inferior infarction. Normal regional values of amplitude and phase were established in 38 individuals without evidence of cardiac abnormality. In 20 patients with anterior infarcts, though the sensitivity of the combined left anterior oblique amplitude and phase images was high (95\%) it was lower (77\%) in 39 with inferior infarcts, principally because the sensitivity of the phase image for the detection of inferior infarcts was only $39 \%$. Right anterior oblique images generated from a first pass study detected all 13 patients with inferior infarcts. The mean left ventricular ejection fraction was significantly lower in the patients with anterior infarcts $(37 \%)$ than in those with inferior infarcts $(48 \%)$. Although the mean wall motion score on $x$ ray contrast ventriculography was slightly lower in the patients with anterior infarction, the high sensitivity of the right anterior oblique amplitude and phase images in inferior infarction suggests that attenuation of counts is an important cause of reduced sensitivity of the left anterior oblique images. This may also partly explain the lower ejection fractions in inferior infarction.

It follows that both a right anterior oblique first pass study and a left anterior oblique equilibrium study are necessary for an accurate description of regional wall motion.

Radionuclide ventriculography permits the noninvasive evaluation of global and regional left ventricular function. Regional wall motion can be assessed by viewing a cine display of the ventriculogram, but the Fourier phase and amplitude images ${ }^{12}$ provide a more objective assessment. ${ }^{3}$ These images quantify the magnitude and timing of count changes in each pixel of the ventriculogram by assuming that the individual pixel activity-time curves approximate to the fundamental frequency of their Fourier transform. The amplitude image can be regarded as showing the extent of wall motion and the phase image as showing the time of minimum counts expressed as a fraction of the cycle. The parametric

Requests for reprints to Dr S R Underwood, Magnetic Resonance Unit, The National Heart and Chest Hospitals, 30 Britten Street, London SW3 6NN.

Accepted for publication 31 May 1988 images show motion of all parts of the left ventricle and they differ from methods that display contours; these only show motion of walls that are perpendicular to the plane of projection. Together, the images detect abnormal wall motion because abnormalities of both the extent and timing of contraction occur after myocardial infarction. ${ }^{45}$

In the usual left anterior oblique projection, each left ventricular pixel represents a narrow column of blood passing from the anterior to the inferior wall. Changes in counts are the result of motion of both of the walls, and abnormal motion of either will lead to abnormalities of amplitude and phase. This may make it unclear which part of the ventricle is giving rise to an abnormality, but in practice the ambiguity can be resolved by the recognition of common patterns of wall motion. For instance, an amplitude defect in the centre of the left ventricle could represent either anterior or inferior hypokinesis, but 

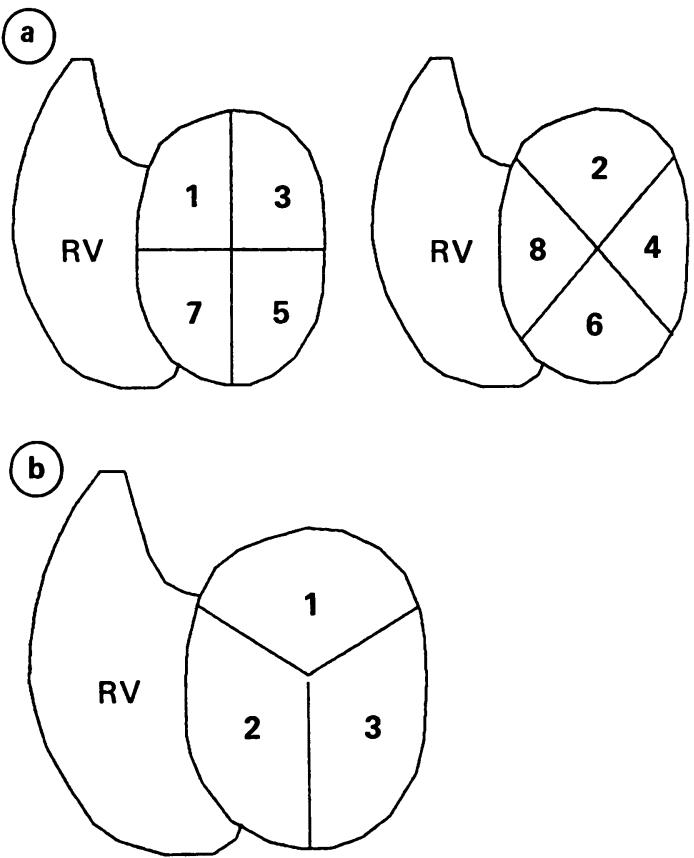

Fig 1 The left ventricle was divided into eight overlapping quadrants in the phase image (a) and three sectors in the amplitude image (b). Mean values were calculated in each region.

anterior hypokinesis is usually associated with septal hypokinesis whereas inferior hypokinesis is not.

A further problem arises from the attenuation of counts from the more distant portion of the blood pool, which means that abnormalities of amplitude and phase in inferior infarction may be small compared with those in anterior infarction. Often there is an amplitude defect in the centre of the left ven-

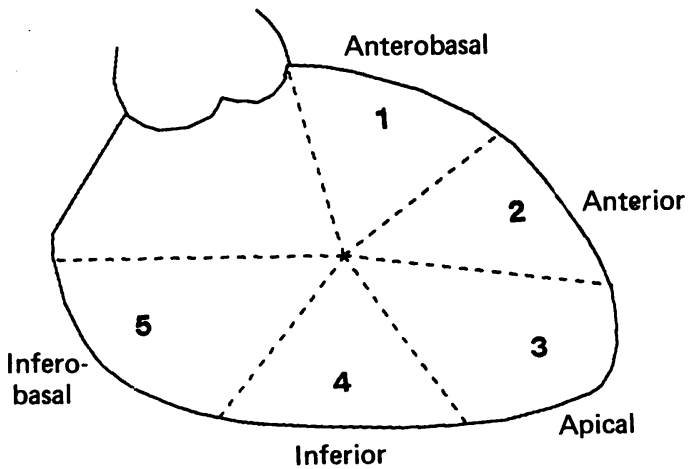

Fig 2 The left ventricular perimeter on the $x$ ray ventriculograms was divided into five segments and wall motion in each was assessed as hyperkinetic (3), normal (2), hypokinetic (1), akinetic (0), or dyskinetic (-1). tricular image, with a normal phase image, although there may be a small area of abnormally high phase at the apex if the inferior infarct is extensive. Small inferobasal infarcts may not be detected at all.

We evaluated the differential sensitivity of amplitude and phase images obtained in the left anterior oblique projection in anterior and inferior infarction and the role of right anterior oblique first pass imaging.

\section{Patients and methods}

Equilibrium radionuclide ventriculography was performed in a left anterior oblique projection with a $30^{\circ}$ caudal tilt after in vivo erythrocyte labelling with $\mathbf{7 4 0}$ $\mathrm{MBq}$ of technetium-99m sodium pertechnetate. We used a General Electric Portacamera IIC with a 200 $\mathrm{mm}$ field of view, interfaced to an Informatek Simmis III computer, to acquire 16 frames per cardiac cycle with electrocardiographic gating. There were 320000 counts per frame and data from cycles that followed one lasting more than $115 \%$ of the mean were rejected. To compensate for the shortfall of counts in the final frame caused by short cycle lengths, each frame was standardised according to the number of cycles contributing to it. Fourier phase and amplitude images were generated after temporal and spatial smoothing. The left ventricular ejection fraction was calculated from count changes within a left ventricular region of interest after automatic background subtraction by means of a functional technique. ${ }^{6}$

First pass radionuclide ventriculography was performed in a right anterior oblique projection before the equilibrium study; the technique we used was described elsewhere. ${ }^{7}$ Data were acquired in list mode and counts obtained during the passage of the bolus through the left heart were reformatted into a representative cine cycle of 16 frames. This usually included eight to twelve cardiac cycles, with 1000 4000 counts within the left ventricle at end diastole. Phase and amplitude images were generated in an identical fashion to the equilibrium study.

To establish normal regional values of amplitude and phase, we studied 38 subjects without evidence of cardiac disease. These were patients being investigated for suspected cardiac disease but in whom coronary arteriography and $x$ ray contrast left ventriculography were normal. Values within two standard deviations of the mean in these subjects for the whole left ventricle and for eight quadrantic regions were regarded as normal (fig 1a). Amplitude was expressed as a percentage of the maximum amplitude within the left ventricle, and normal values were established for the whole ventricle and for three $120^{\circ}$ sectors, representing the base, the anteroseptal wall, 
Table 1 Characteristics of the groups of patients with anterior and inferior infarcts

\begin{tabular}{lll}
\hline Characteristic & Anterior & Inferior \\
\hline Total number & 20 & 39 \\
Age (range) & $52(36-70)$ & $54(37-73)$ \\
Sex & $19 \mathrm{M}, 1 \mathrm{~F}$ & $36 \mathrm{M}, 3 \mathrm{~F}$ \\
ECG findings: & & 0 \\
Anterior or anteroseptal Q wave & 16 & 35 \\
Inferior or inferolateral Q wave & 2 & 2 \\
LBBB & 0 & 2 \\
Normal & 2 & 30 \\
Extent of disease: & 14 & 4 \\
Three vessel disease & 3 & 5 \\
Two vessel disease & 3 & $34(1)$ \\
One vessel disease & $20(16)$ & $30(2)$ \\
Vessels diseased (occluded): & $17(0)$ & $39(23)$ \\
LAD & $14(4)$ & \\
LCx & &
\end{tabular}

ECG, electrocardiogram; LAD, left anterior descending; LCx, left circumflex; RCA, right coronary artery; LBBB, left bundle branch block.

and the posterolateral wall (fig $1 \mathrm{~b}$ ).

Thirty nine patients with a single transmural inferior myocardial infarct and 20 with a single transmural anterior infarct were studied by radionuclide ventriculography (table 1 ). An equilibrium study in the left anterior oblique projection was performed in all patients, and a first pass study was performed in the right anterior oblique projection in 13 of the patients with inferior infarction. In each patient, mean regional amplitude and phase in the left anterior oblique images were compared with the results in patients without cardiac disease, and mean values in the right anterior oblique images were compared with previously established normal ranges.'

Infarction was diagnosed on the grounds of history, electrocardiographic changes, and abnormal wall motion on a right anterior oblique $x$ ray contrast ventriculogram. The reasoning behind selection was to obtain two groups with abnormal motion that was as nearly as possible restricted to the anterior or the inferior left ventricle. Patients with $Q$ waves in only the septal or the lateral leads were excluded, but those with $\mathbf{Q}$ waves in the septal or lateral leads in addition to the anterior or inferior leads were included. An experienced cardiologist who was not aware of the radionuclide findings analysed the $x$ ray ventriculograms. The perimeter of the left ventricle was divided into five equal sectors representing the anterobasal, anterior, apical, inferior, and inferobasal areas (fig 2). In each area, wall motion was classified as hyperkinetic, normal, hypokinetic, akinetic, or dyskinetic. Patients with equal grades of abnormality of both the anterior and inferior walls were excluded, but patients with akinesia or dyskinesia of the anterior wall and hypokinesia of the inferior wall were included in the anterior group and vice versa.
Patients with abnormalities in the apical sector were included in the anterior or inferior group according to whether adjoining abnormalities were present in the anterior or inferior wall.

\section{Results}

Table 2 shows the values for amplitude and phase in the normal individuals. There were no regional variations in phase, but mean basal amplitude was significantly lower than the global value $(p<0.01)$ because of the overlap between the left atrium and ventricle. Amplitude in the septum was lower than in the lateral wall $(p<0.01)$.

Table 3 shows the wall motion scores in the two groups of patients. The mean total wall motion score was slightly lower in those with anterior infarcts than in those with inferior infarcts, but the difference was not statistically significant ( $p>0.05$ by Student's $t$ test). The main regional abnormalities were in the anterior and apical regions (2 and 3 ) in anterior infarction, and in the inferior and inferobasal regions (4 and 5) in inferior infarction.

Mean (SD) left ventricular ejection fraction in the group with anterior infarcts was $37.2(18.8) \%$ and $48.0(14.3) \%$ in the group with inferior infarcts $(p<$ $0.05)$. Seventeen patients $(85 \%)$ in the group with anterior infarcts and 23 patients $(59 \%)$ in the group with inferior infarcts had abnormal ejection fractions $(<50 \%)$.

Table 2 Normal mean (SD) of amplitude and phase in the whole left ventricle ( $L V)$, and in the three regions shown in fig $1 b$ for amplitude and the eight regions shown in fig $1 a$ for phase

\begin{tabular}{lcccccccccc}
\hline & $L V$ & 1 & 2 & 3 & 4 & 5 & 6 & 7 & 8 \\
\hline Amplitude: & & & & & & & & & \\
Mean (\%) & 58 & 51 & 55 & 62 & - & - & - & - & - \\
SD (\%) & 5.0 & 6.9 & 4.2 & 5.1 & - & - & - & - & - \\
Phase: & & & & & & & \\
Mean $\left(\left(^{\circ}\right)\right.$ & 135 & 136 & 134 & 136 & 136 & 133 & 135 & 136 & 138 \\
SD $\left({ }^{\circ}\right)$ & 14 & 15 & 14 & 14 & 14 & 15 & 15 & 15 & 15 \\
\hline
\end{tabular}

The amplitude values are expressed as percentage of the maximum value in the left ventricle.

Table 3 Mean total and regional wall motion scores from $x$ ray contrast ventriculography in the patients with anterior and inferior infarcts

\begin{tabular}{|c|c|c|c|c|c|c|}
\hline & Total & 1 & 2 & 3 & 4 & 5 \\
\hline $\begin{array}{c}\text { Anterior: } \\
\text { Mean } \\
\text { SD } \\
\text { Inferior: }\end{array}$ & $\begin{array}{l}4 \cdot 9 \\
2 \cdot 24\end{array}$ & $\begin{array}{l}1 \cdot 9 \\
0.30\end{array}$ & $\begin{array}{l}0.3 \\
0.68\end{array}$ & $\begin{array}{l}0.1 \\
1.02\end{array}$ & $\begin{array}{l}0.9 \\
0.86\end{array}$ & $\begin{array}{l}1.7 \\
0.43\end{array}$ \\
\hline $\begin{array}{l}\text { Mean } \\
\text { SD }\end{array}$ & $\begin{array}{l}5.4 \\
1.95\end{array}$ & $\begin{array}{l}1.9 \\
0.35\end{array}$ & $\begin{array}{l}1.5 \\
0.63\end{array}$ & $\begin{array}{l}1 \cdot 2 \\
1 \cdot 10\end{array}$ & $\begin{array}{l}0.5 \\
0.62\end{array}$ & $\begin{array}{l}0.3 \\
0.49\end{array}$ \\
\hline
\end{tabular}

Regions 1 to 5 correspond to the anterobasal, anterior, apical, inferior, and inferobasal regions respectively (fis 2). The scores were assigned as: 3 , hyperkinesis; 2 , normal; 1, hypokinesis; 0 , akinesis; -1 , dyskinesis. 

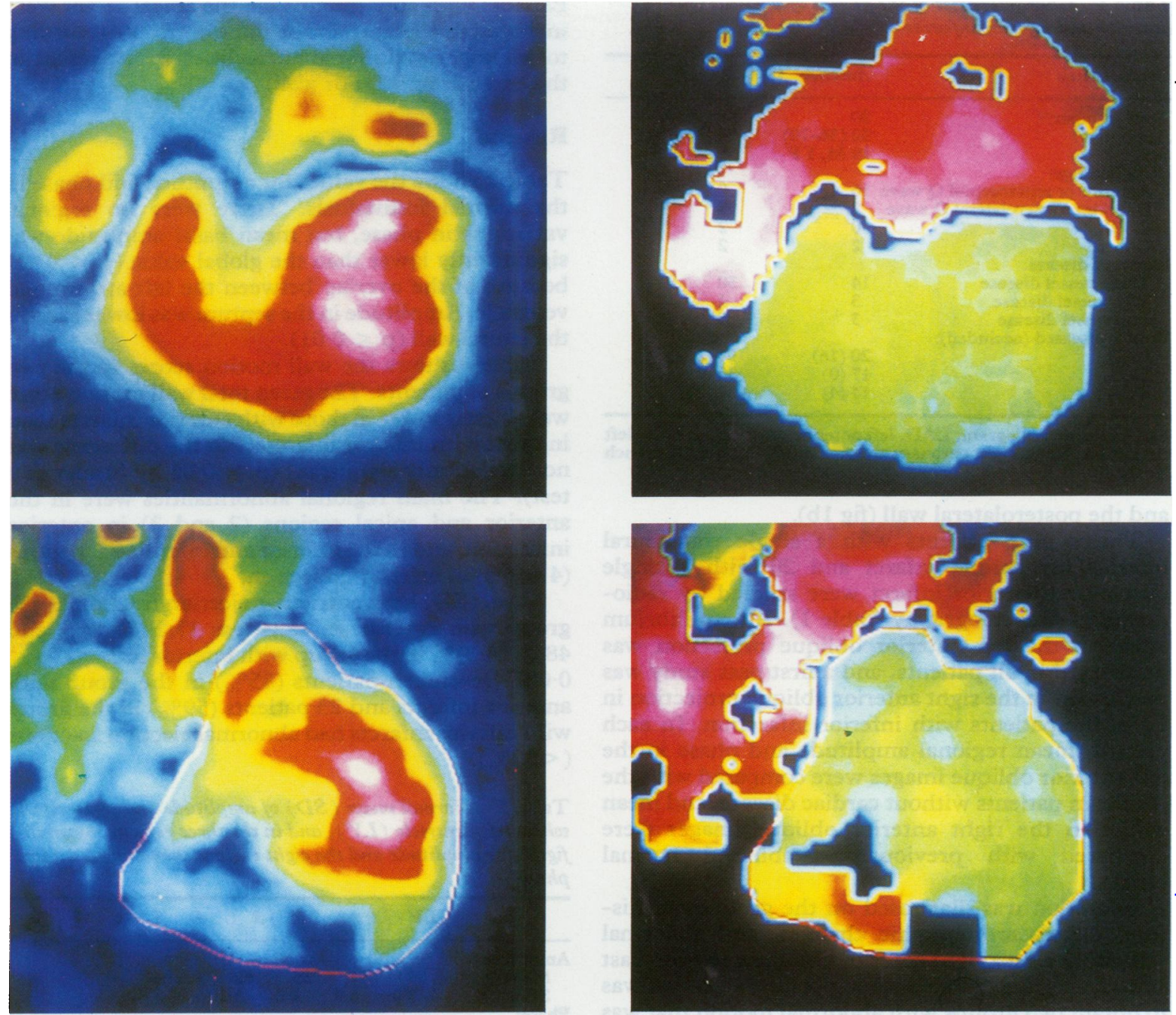

Fig 3 Left anterior oblique (top) and right anterior oblique (bottom) amplitude (left) and phase images (right) in a patient with inferior infarction. The normal ventricular phase is green and there should be high values of amplitude within the left ventricle (red). The left anterior oblique images are normal apart from a slight reduction in lateral amplitude. The right anterior oblique images show inferobasal abnormalities.

Table 4 Numbers (\%) of patients in the groups with anterior and inferior infarcts with abnormalities of the amplitude and phase images seen separately and in either the amplitude or the phase image obtained in the left anterior oblique projection

\begin{tabular}{llll}
\hline & Amplitude & Phase & Amp + phase \\
\hline Anterior & $16(80)$ & $19(95)$ & $19(95)$ \\
Inferior & $26(67)$ & $19(39)$ & $30(77)$ \\
\hline
\end{tabular}

Table 4 shows the numbers and percentages of patients with abnormalities in the amplitude and $N$ phase images obtained in the left anterior oblique projection. The images were more sensitive detectors of anterior infarction than of inferior infarction. The $\chi^{2}$ test showed a significant difference in sensitivity $\stackrel{S}{\rightarrow}$ for the phase image alone $(p<0.001)$ but not for the $T$ amplitude image or for the two images combined (p > 0.05).

The amplitude image obtained in the right anterior 
Table 5 Numbers (\%) of patients with inferior infarcts (of the 13 studied in two projections) who had abnormalities of the amplitude and phase images in the left and right anterior oblique projections

\begin{tabular}{lccc}
\hline & Amplitude & Phase & Amp + phase \\
\hline Left anterior oblique & $7(54)$ & $4(31)$ & $7(54)$ \\
Right anterior oblique & $13(100)$ & $11(85)$ & $13(100)$ \\
\hline
\end{tabular}

oblique projection was abnormal in all 13 patients studied in both projections and the phase image was abnormal in 11 (table 5). The $\chi^{2}$ test showed a significant difference in sensitivity between right anterior oblique and left anterior oblique images for the detection of inferior infarction ( $p<0.001$ for amplitude and $p<0.01$ for phase).

Figure 3 shows the findings in a patient with inferior infarction. The amplitude and phase images in the left anterior oblique projection are normal, but the right anterior oblique images both show abnormalities of amplitude and phase that are typical of infarction of the inferior wall.

\section{Discussion}

The results show that amplitude and phase images obtained in the left anterior oblique projection are highly sensitive $(95 \%)$ detectors of anterior infarction but that they are less sensitive $(77 \%)$ detectors of inferior infarction. Most of the loss of sensitivity was the result of the phase image, which was abnormal in $95 \%$ of patients with anterior infarction but in only $39 \%$ of those with inferior infarction. Because an abnormal value was defined as one that was more than two standard deviations from the normal mean, the specificity for each test was $97 \%$.

There are two possible reasons for the reduced sensitivity. Attenuation of counts from the more distant inferior portion of the blood pool means that motion of the inferior wall of the left ventricle contributes less to changes in counts seen in each pixel. For a ventricle of average size the attenuation of counts from the inferior wall is approximately three times greater than that from the anterior wall. An alternative explanation is that the abnormalities of wall motion were less severe in the group with inferior infarcts. Global wall motion score in anterior infarction was less than in inferior infarction and though the difference was not statistically significant, there was a significant difference $(p<0.05)$ between the two regions with the main abnormalities (anterior and apical for anterior infarction, and inferior and inferobasal for inferior infarction). Attenuation does not differ in the right anterior oblique projection, however, and because the right anterior oblique images were abnormal in every case of inferior infarction, attenuation must make a considerable contribution to the reduced sensitivity. If the severity of the abnormality of wall motion were the only explanation, then the amplitude and phase images would have been expected to be equally sensitive in the two projections.

It is difficult to explain why the sensitivity of the phase image is less than that of the amplitude image in inferior infarction. If two similar curves with different amplitude are summed, the resultant amplitude is equal to the mean amplitude of the components. Phase is a more complex variable, however, and it does not follow that summing two curves with different phase produces a curve with phase between that of the components. It is possible that a volume curve with high phase and low amplitude from the inferior portion of the ventricle, added to a normal curve from the anterior portion, results in a curve with reduced amplitude but normal phase.

Attenuation will also affect the calculation of ejection fraction and this has been shown in phantom studies by Schneider $e t$ al.$^{8}$ In patients with previous infarction, Reduto et al reported a mean left ventricular ejection fraction of $34 \%$ after anterior infarction and one of $50 \%$ after inferior infarction, measured by radionuclide ventriculography'; these values are similar to the $37 \%$ and $48 \%$ in this study. A lower ejection fraction after inferior infarction has also been described in $x$ ray ventriculography, ${ }^{10}$ but in view of the findings in this study, at least part of the difference must be because the radionuclide volume curve is weighted by counts from the anterior portion of the ventricle.

We found that amplitude and phase images obtained in the left anterior oblique projection were very sensitive detectors of anterior infarcts but less so of inferior infarcts. Much of the reduced sensitivity arose from the phase image. Right anterior oblique images were very sensitive detectors of both anterior and inferior infarcts. The findings can be accounted for by a combination of attenuation of counts from the inferior portion of the blood pool and less severe wall motion abnormalities in inferior infarction. The lower ejection fraction in anterior infarction is also partly the result of attentuation, and radionuclide ejection fraction is overestimated in inferior infarction. We believe that a combination of a right anterior oblique first pass study and a left anterior oblique equilibrium study gives the most reliable assessment of regional wall motion.

\section{References}

1 Geffers H, Adam WE, Bitter F, Seigel H, Kampmann H. Data processing and functional imag- 
ing in radionuclide ventriculography. In: Brill $\mathbf{A B}$, Price RR. Information processing in medical imaging. Proceedings of the 5th international conference. Nashville: Biomedical computing technology information center, 1977:322-32.

2 Adam WE, Tarkowska A, Bitter F, Stauch $M$, Geffers H. Equilibrium gated radionuclide ventriculography. Cardiovasc Radiol 1979;2:161-73.

3 Walton S, Yiannikas J, Jarritt PH, Brown NJG, Swanton RH, Ell PJ. Phasic abnormalities of left ventricular emptying in coronary artery disease. $\mathrm{Br}$ Heart J 1981;46:245-53.

4 Botvinick E, Dunn R, Frais M, et al. The phase image: its relationship to patterns of contraction and conduction. Circulation 1982;65:551-60.

5 Cardot JC, Berthout P, Verdenet J, et al. Temporal Fourier analysis applied to equilibrium radionuclide cineangiography. Eur J Nucl Med 1982;7:353-8.

6 Goris ML, Briandet PA, Huffer E. Automation and operator independent data processing of cardiac and pulmonary functions: role, methods, and results. In:
Di Paolo R, Kahn E, eds. Information processing in medical imaging. Proceedings of the 6th international conference. Paris: INSERM, 1979:88:427-48.

7 Walton S, Ell PJ, Jarritt PH, Swanton RH. Phase analysis of the first pass radionuclide angiocardiogram. Br Heart J 1982;48:441-8.

8 Schneider RM, Jaszczak RJ, Coleman RE, Cobb FR. Disproportionate effects of regional hypokinesis on radionuclide ejection fraction: compensation using attenuation-corrected ventricular volumes. $J$ Nucl Med 1984;25:747-54.

9 Reduto LA, Berger HJ, Cohen LS, Gottschalk A, Zaret BL. Sequential radionuclide assessment of left and right ventricular performance after acute transmural myocardial infarction. Ann Intern Med 1978;89:441-7.

10 Miller RR, Olsen HG, Vismara LA, Bogren HG, Amsterdam EA, Mason DT. Pump dysfunction after myocardial infarction: importance of location extent and pattern of abnormal segmental left ventricular contraction. Am J Cardiol 1976;37:340-4. 